PRAXIS

ecucativa

Universidad Nacional de La Pampa

Facultad de Ciencias Humanas

Instituto de Ciencias de la Educación

para la investigación interdisciplinaria

\section{4 米当战 \\ Instituto de Ciencias de la Educación

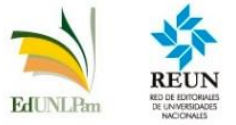

ISSN 2313-934X

SANTA ROSA, LA PAMPA, ARGENTINA

Correo electrónico: iceii@humanas.unlpam.edu.ar

Disponible en https://cerac.unlpam.edu.ar/index.php/praxis

Historia de las mujeres y género en el nivel universitario. Acerca del Profesorado en Historia, de la Universidad Nacional de La Plata. Artículo de Milagros Rocha Praxis educativa, Vol. 24, No 1 enero - abril 2020 - ISSN 2313-9334X. pp. 1-15. DOI: https://dx.doi.org/10.19137/praxiseducativa-2020-240106

Historia de las mujeres y género en el nivel universitario. Acerca del Profesorado en Historia, de la Universidad Nacional de La Plata

History of women and gender at the university level. About the History Teacher, of the National University of La Plata

História das mulheres e gênero no nível universitário. Sobre o professor de História, da Universidade Nacional de La Plata

\title{
Milagros Rocha
}

Facultad de Humanidades y Ciencias de la Educación, Universidad Nacional de La Plata-

Argentina

milagrosmrocha@gmail.com

ORCID 0000-0002-5361-0211

Recibido: 2019-08-19 | Revisado: 2019-15-10 | Aceptado: 2019-20-10

\section{Resumen}

Los estudios de género y mujeres han cobrado mayor visibilidad en los últimos años. Un avance tangible no sólo en términos académicos, sino también a nivel social y educativo. Si bien en nuestro país estas temáticas han ganado notoriedad, observamos que esto no repercute con la misma intensidad en el ámbito educativo. En el nivel secundario estos temas ingresan preferentemente de la mano de la Ley de Educación Sexual Integral (Ley № 26.150) aunque esto no ocurre de manera sistemática. Nos preguntamos entonces por la Historia que se enseña no sólo en secundaria, sino también en la Universidad. En este marco, puntualmente indagamos el ingreso 
de estas temáticas dentro del Profesorado en Historia, de la Universidad Nacional de La Plata. En suma, un escrito que pretende problematizar qué sujetos históricos recobran voz en la enseñanza de la Historia universitaria y a la vez presenta al currículum como artefacto cultural, histórico y generizado.

Palabras claves: Historia, mujeres, currículum, formación, Universidad.

Abstract

Gender and women's studies have become more visible in recent years. A tangible advance not only in academic terms, but also at a social and educational level. Although these issues have gained notoriety in our country, we note that this does not affect the same intensity in the educational field. At secondary level, these issues enter preferably from the hand of the Comprehensive Sexual Education Law (Law No. 26,150) although this does not happen systematically. We ask ourselves then about the History that is taught not only in high school, but also at University. Within this framework, we promptly inquire into the entry of these issues within the History Teachers Program at the National University of La Plata. In sum, this is a paper that tries to question which historical subjects regain voice in the teaching of university History and at the same time it presents the curriculum as a cultural, historical and gendered device.

Keywords: History, women, curriculum, training, university.

\section{Resumo}

Estudos sobre gênero e mulheres ganharam maior visibilidade nos últimos anos. Um avanço tangível não apenas em termos acadêmicos, mas também em nível social e educacional. Embora esses temas tenham ganhado notoriedade em nosso país, notamos que isso não afeta a mesma intensidade no campo educacional. No nível secundário, essas questões de preferência entram na mão da Lei de Educação Sexual Abrangente (Lei No. 26.150), embora isso não ocorra de forma sistemática. Perguntamo-nos então sobre a História que é ensinada não apenas no ensino médio, mas também na universidade. Dentro deste quadro, nós prontamente investigamos a entrada destes temas dentro do corpo docente em História, da Universidade Nacional de La Plata. Em suma, um trabalho que procura problematizar quais sujeitos históricos recuperam a voz no ensino da história universitária e, ao mesmo tempo, apresenta o currículo como artefato cultural, histórico e gerado.

Palavras chaves: história, mulheres, currículo, treinamento, universidade.

En los últimos años, impulsadas por un creciente y cada vez más visible movimiento feminista, han aumentado las demandas sociales en contra de las violencias sexistas: las masivas marchas por \#NiUnaMenos desde 2015, la convocatoria al Paro Internacional de Mujeres (\#8M) a partir de 2017 y la concurrida celebración del 34을 Encuentro Nacional de Mujeres, en la ciudad de La Plata, en octubre de 2019. Estas, entre otras movilizaciones, ponen en agenda la necesidad de visibilizar y dar lugar a los reclamos. Claro está que el movimiento feminista no es nuevo y su vinculación con el ámbito académico tampoco. Ahora, cómo dialogan estas temáticas con el ámbito educativo, qué incidencia tienen estos temas en los niveles educativos; por otra parte, cuál es la situación en las diversas regiones de nuestro país. Responder tales interrogantes es un horizonte deseable. Por lo pronto, en estas líneas, se desarrollan algunos de los resultados de mi tesis de maestría en relación con el nivel universitario, puntualmente con el Profesorado en Historia de la Universidad Nacional de La Plata.

En suma, este artículo pretende historizar cómo se fue configurando y haciéndose visible el ingreso de la Historia de las mujeres y género en dicha carrera, así como en la propia Facultad de Humanidades y Ciencias de la Educación (FaHCE). Esto se sustenta a 
partir de entrevistas realizadas a diversos actores claves de la Unidad Académica, el análisis del currículum estructural-formal (planes de estudios y programas) y voces docentes de la carrera. En definitiva, un campo fértil por explorar y una apuesta por comprender el currículum en tanto artefacto cultural, histórico, situado y generizado.

Puntos que hacen trama: mujeres en la "historia enseñada" universitaria

Explorar la enseñanza de la Historia de las mujeres y género, en el Profesorado de Historia de la Universidad Nacional de La Plata, nos lleva a señalar una tradición de temáticas vinculadas, esencialmente a las mujeres, aunque también emergen casos puntuales que profundizan aspectos relacionales. Estos antecedentes los hallamos, principalmente, en las materias denominadas "Materias Problemas". Estas asignaturas se crean a partir del Plan de Estudios 93 a fin de ahondar en el estudio de una problemática de las áreas de Historia Argentina y Americana. Sin embargo, encontramos un antecedente a destacar: nos referimos a un programa de seminario dictado en 1989, por la Profesora Silvia Mallo, titulado "La mujer en el período colonial". En dicho documento, se especifica: "se considera necesario en este tipo de seminarios no sólo establecer la validez y entidad del tema como posible área histórica a investigar, sino también la búsqueda de diferentes formas metodológicas para abordar el mismo" (Mallo, 1989, p. 1). Los contenidos versan entre:

La mujer como temas de estudios históricos, historia de las mujeres o la mujer en la historia. La mujer en la sociedad tradicional y moderna. Proyección en la Historia Argentina. Familia y mujer. La cuestión de las esferas de acción masculina-femenina. Hogar y unidad doméstica. Matrimonio, hijos, educación y salud. El honor y la religiosidad como distintivos femeninos. Mitos. La brujería femenina. La mujer en la legislación occidental. El papel de la mujer en el Estado. Mujer y poder. Las formas de participación política de la mujer, entre otros contenidos (Mallo, 1989, p. 1 y 2).

Asimismo, el programa explicita que asistirán especialistas invitados para compartir sus investigaciones. Aparecen nombrados allí destacados profesores de esta casa de estudios como Carlos Mayo, convocado para hablar sobre "Robo de mujeres y relaciones extramatrimoniales en la campaña" (siglo XVIII) y Osvaldo Barreneche, para presentar la temática "Violencia y criminalidad femenina".

Durante el debate del nuevo y vigente Plan de Estudio (2011) hubo disputas, revisiones y algunos reclamos del estudiantado que tenían que ver con incorporar temáticas. La demanda se concentró no en una perspectiva de género, sino en el ingreso de la historia reciente (ya que uno de los reclamos era que la materia Historia Argentina II no llegaba a abordarlo). En el marco de dicha indagación, se elaboraron nueve entrevistas a personas que ocuparon cargos relevantes en la vida institucional, ${ }^{i}$ tanto en la actualidad como hace unos años. En una de las entrevistas, se expresa: "el Plan 93 tenía la gran ventaja de establecer la existencia de las Materias Problema, entonces muchas de las demandas de los alumnos se podían incorporar como Materia Problema" (Fragmento de entrevista) Se menciona que, de esta manera, se pudieron incorporar materias que se ocupaban de distintos aspectos de la historia reciente en Argentina y en Latinoamérica y que, además de haber bastante investigación sobre historia reciente, el Departamento contaba con docentes con formación. Asociado a estos argumentos, el mismo entrevistado revela que:

Las Materias Problemas servían, también, para incorporar algunas cuestiones historiográficas que no estaban incorporadas, y yo me acuerdo que una de las cuestiones que se pudieron incorporar era la Historia del medioambiente, y la segunda, y te digo sin que hubiera una demanda en principio previa, fue la historia de género (Fragmento de entrevista). 
Por otra parte, la entrevista aporta otro argumento a favor de las Materias Problema, pues, más allá de capitalizarse un espacio donde poder acoger las demandas estudiantiles, estas, a su vez, contribuyeron a generar espacios de inserción laboral para docentes:

Entonces eso fue como una válvula de escape para poder ocupar, darles un espacio a esos jóvenes y que la mayoría de éstos después concursaron en las cátedras (...) gente muy brillante, muy preparada y que a partir de ahí pudo ocupar un lugar distinto dentro del Departamento. Bueno, y así se incorporó [2005] a IndiValobra. (Fragmento de entrevista).

Esto nos lleva a considerar que no necesariamente la creación de espacios vinculados a temáticas de género y/o mujeres respondió a demandas estudiantiles 0 sociales. En este caso, el testimonio nos brinda otro elemento de análisis al exponer que la creación de algunas Materias Problema respondió a otras preocupaciones vinculadas a absorber un potencial de docentes formados en ciertas temáticas "novedosas" o relevantes para la política educativa del Departamento, aunque no eran solicitadas en ese momento como centrales por el estudiantado y que, asimismo, eran temas que ganaban cada vez más lugar en el campo disciplinar. Las Materias Problema han aportado variabilidad y amplitud a las temáticas ofrecidas por el Departamento: mujer, familia, sexualidad, feminismo, cuerpos, "mundo de hombres", matrimonio y masculinidades. En función del relevo de programas," se observa que varias de estas materias tienen como denominador común preocupaciones situadas en el siglo XIX. Asimismo, no todas presentan en su título una referencia a la temática en cuestión, aunque guardan temas afines, como en el caso del programa de Cabanillas que presenta un cruce entre decolonialidad y género (2016). Un caso semejante sucede con los programas de la profesora Aguirre (2015 y 2017) y Troisi (2009) dentro de los cuales emergen contenidos y lecturas afines. Finalmente, los cursos dictados por Valobra desde 2005, de manera continua y sostenida, se posicionan desde una perspectiva de mujeres y género con anclaje en la teoría feminista, aportando, en 2017, el primer seminario exclusivamente sobre masculinidades en la carrera de Historia.

De lo expuesto, se señalan dos aspectos. Por un lado, en relación a la Facultad de Humanidades y Ciencias de la Educación (UNLP), consignar que las transformaciones curriculares se inscriben próximas a cuestiones de género: un centro de investigación creado en 2007 (Centro Interdisciplinario en Investigaciones en Género, CInIG), una carrera de posgrado con formación específica en esta temática establecida en 2012 (Especialización en Educación en Géneros y Sexualidades), el Protocolo de actuación ante situaciones de discriminación y/o violencia de género, en 2015, sustentado en los principios que sostiene el Programa contra la violencia de género de la UNLP; la organización y configuración de una cátedra nueva de carácter optativa, en 2016 (Introducción a la teoría feminista y de género), por su parte, mencionamos las políticas de ingreso en donde, a partir de 2018, se implementaron talleres sobre género. Respecto a la vida institucional, indicamos la disposición de baños universales, señalamos a su vez la modificación, en 2017, del Régimen de Enseñanza y Promoción (REP) en donde se reconocen los usos plurales y dinámicos del lenguaje. A excepción del CInIG, creado en 2007, la mayoría de estas implementaciones o transformaciones se inscriben en un contexto relativamente reciente, estableciendo un camino en paralelo, y en diálogo, con las demandas sociales. Por otro lado, respecto a la carrera de historia, emerge con mayor notoriedad la visibilidad de la mujer en la historia más que una mirada generizada en términos más amplios de modo que involucre una inflexión sobre las relaciones de género y el modo en que se construyen identidades de género en plural. Asimismo, en este relevo de documentos, el estudio de temáticas sobre mujeres y género en la carrera antecede a este reclamo social que se hace más notorio a partir de la primera marcha $\mathrm{Ni}$ 
una menos, en 2015. Si bien estos hechos inciden como campo de determinación, hay que indicar que la carrera ofrecía con anterioridad espacios de estudios afines de la mano de las Materias Problemas, aunque sin duda se evidencia, hace aproximadamente tres años, una mayor visibilización y problematización de estas temáticas.

\section{Enseñar Historia de las mujeres y género: ¿campo específico o campo general?}

En relación con la enseñanza de la Historia de las mujeres y género, en la tesis planteamos una disyuntiva respecto a pensarla (y enseñarla) como campo general y/o campo específico. En esta clave, en una de las entrevistas, se alude a que en el Departamento de Historia:

Ha habido una especie de implosión en varios sentidos, desde ya de la producción académica, en donde uno empieza a conocer más. Uno en el Departamento, inmediatamente, conoce qué profesores son los que se especializan en ciertas temáticas y [ello es así], particularmente, en el caso de la temática de género. Pero lo cierto es que, de un tiempo a esta parte, ha empezado a surgir una necesidad, en varias cátedras, que por ahí no tenían esa formación; una necesidad de empezar a incorporar en las propias currículas de las cátedras este tema en particular (Fragmento de entrevista).

En primer lugar, destacamos la idea de implosión en la producción académica historia investigada - lo cual nos habla de un desmoronamiento de las narrativas historiográficas hegemónicas (D’Antonio, 2012, 2013) y el hundimiento de esas posiciones en el propio campo.

En relación con el dilema de campo específico o general, en una de las entrevistas se lleva esta disyuntiva a otros espacios formativos como son los Congresos y Jornadas. Vinculado a esto, se plantea que resulta frecuente encontrar investigaciones sobre cualquier tema que ignoran la producción de género y mujeres porque lo consideran algo particular, reproduciendo de esta manera el binarismo y el esencialismo particularista que hay respecto de las mujeres o de cualquier persona cuya cuestión de género no sea estudiado desde la masculinidad hegemónica:

Vos podés encontrar un estado de la cuestión donde no te citan, aunque están trabajando, por ejemplo, peronismo, porque bueno [se considera que] "vos hacés un aporte particular", (...) [pero después] aparecen cosas "[tan] generales" como el sindicato del papel en Ushuaia durante el período peronista, y eso no es [considerado] particular, eso es [considerado] general (Fragmento de entrevista) ${ }^{i i i}$. Expresa que esto no ha cambiado, lamentablemente, y que había propuesto una idea un tanto impactante por su paralelismo, "el ghetto del género":

Ghetto en el que nos encierran y nos encerramos, porque qué pasa: vos vas a un congreso <general>, a una mesa "general" y, entonces, vos expones tu trabajo sobre género y todos te ven como algo "particular" que no tiene mucho que ver con la mesa y que no saben muy bien cómo engancharlo con lo que están discutiendo (Fragmento de entrevista).

$Y$ agrega que, justamente, lo interesante de una historia de las mujeres con perspectiva de género es poder reformular las interpretaciones existentes a la luz de un sujeto distinto del que se mira, que es un sujeto masculino (Haraway, 1995). Sin embargo, manifiesta que esto no cala y que es difícil salir de esa mirada de que "ofreces algo particular". Esto nos coloca frente a los dilemas que, en términos conceptuales, reponen tanto Carole Pateman (1995) como Joan Scott (1996) con sus paradojas. En efecto, las mujeres y los estudios de género reclaman un lugar desde una particularidad, pero no por ello, dejan de exigir reconocimiento en igualdad de condiciones que otrxs sujetxs. Es irresoluble y es en esa tensión que se mueven los aportes disciplinares. 
En la actualidad, no solo hay personas referentes en el tema, sino que también comienza a expandirse el interés sobre mujeres y género en varias cátedras. Esto da origen, según uno de los testimonios, a problemáticas vinculadas a la especialidad, enseñanza y sensibilidad personal con el tema:

Esa idea de pensar en esta división entre los "especialistas" y "los que no" ha sido una barrera, me parece, muchas veces, que ha tendido más a la comodidad de quienes dicen: "este tema no entra en nuestra currícula". Porque, en definitiva, uno no es especialista y me parece que un poco de la mano de los movimientos de mujeres, de los reclamos que hoy empiezan a ser cada vez más cotidianos y demás, ha habido una necesidad de romper con esa comodidad (Fragmento de entrevista).

Este fragmento retoma la idea de que surge de las preguntas con relación a cómo ingresa la Historia de las mujeres y género como ¿campo general o campo específico? Resulta interesante reparar en la reflexión en torno a la "comodidad" o, también, como contracara, podríamos pensar en la posible dificultad que puede llegar a generar la inclusión de estos temas. Así, otro testimonio entiende que las discusiones que se dieron en las reuniones de junta tienen que ver con visibilizar la problemática de género dentro de la carrera, y arguye que "desoír hoy la ESI es ya un posicionamiento". Esto indica que dicha temática se convierte en algo instituyente que interpela, desde diversos ámbitos y actores institucionales, lo instituido. Asimismo, expresa algo semejante a lo que observa el testimonio anterior respecto de lo etario. En los últimos años, ha habido un giro, sobre todo por la incorporación a través de selecciones y concursos docentes, de camadas de profesores y profesoras cada vez más jóvenes, que ingresan a las cátedras con inquietudes o perfiles de investigación vinculados a la temática de género y mujeres. Manifiesta que ese nuevo plantel docente, por lo general, se ha hecho eco por cuestiones personales o de agenda, incorporando textos, en la metodología de sus clases, en donde tienden a visibilizar estos temas. De todos modos, enuncia que estas temáticas siguen siendo, dentro de la carrera, una porción muy marginal del Plan de Estudios o generándose actividades en espacios no formales de la currícula ${ }^{\text {iv }}$ y que, si bien hay materias o seminarios optativos, eso significa que la formación depende entonces, de la inquietud personal del que se sienta atravesado por estos temas. Esa disposición personal no solo implica formación académica para la modificación de programas, búsqueda de bibliografía, armado de clase y demás, sino también embarcarse en otras discusiones, "comprendiendo que la identidad del/la docente constituye una 'herramienta' en el propio proceso, con frecuencia en formas incómodas o impredecibles" (Flores, 2015, p. 8).

Pensar la enseñanza de estas temáticas requiere un ejercicio de deconstrucción para que las mismas no queden relegadas a una "clase especial" o Materia Problema optativa, o a cargo, solamente, de referentes a partir de intereses personales y académicos.

Enseñar historia con mujeres y perspectiva de género

En el reglamento de los Planes de Estudios de la Universidad Nacional de La Plata (Ordenanza № 282/10), " se detalla la estructura de presentación e información que requiere un Plan de Estudio. El ítem 13 menciona los "objetivos de formación y contenidos de cada espacio curricular". Este ordenamiento requiere nombre de la asignatura, régimen de cursada, carga horaria semanal, carga horaria total, objetivos y contenidos mínimos. En estos últimos, nos centraremos para comenzar a analizar qué sucede con esta historia investigada en estas expresiones "mínimas" de contenidos.

En líneas generales, podemos señalar que prima una mirada masculina naturalizada en los contenidos de las materias troncales, una universalidad devenida en 
arbitraria y masculina (Scott, 2003) fuertemente atravesada por un análisis "clasista" o materialista. Como señalamos antes, retomando a Débora D’Antonio (2012, 2013), la intersección entre historia social, clase y género, en el plano historiográfico nacional, ha tenido (por el momento) un bajo impacto en las narrativas historiográficas hegemónicas como así también en la historia enseñada. En cierta forma, el currículum se presenta colonizado por la masculinidad en tanto también la historiografía lo estaba o no recogía los resultados de los aportes de la Historia de las mujeres o género (historia investigada).

Por ello, en términos comparativos, los contenidos mínimos del Plan 2011, solo en algunas materias, ampliaron la mirada de Ixs sujetxs sociales intervinientes en la historia. La temática en torno a las mujeres y género ingresa explícitamente en los contenidos mínimos de dos cátedras (Introducción a la Historia e Historia General V), lo cual contrasta respecto del Plan anterior (1993). Asimismo, se alude, en la materia Historia Argentina II, a la "invisibilización de las comunidades indígenas" (2011, p. 12). Así, la visibilización (y diversificación) de agentes históricos en la enseñanza de la historia obligatoria de la carrera se encuentra en movimiento.

Ahora bien, problematizamos a su vez las intenciones que subyacen detrás de un Programa de cátedra, es decir, daremos lugar a las voces docentes en tanto voces que portan saberes construidos en y desde la experiencia, como expresa Edelstein, "conocimientos profesionales que se construyen en el marco de los procesos de intervención (...) posible desde una posición de reflexividad crítica contextualizada" (2015, p. 211). En el marco de la tesis de Maestría, se elaboró, como herramienta de consulta e indagación, un formulario que fue enviado a docentes que dictan estas materias obligatorias. Sobre un total de sesenta formularios emitidos recibimos cuarenta respuestas. En esta oportunidad, nos interesa tomar de referencia la primera pregunta de este instrumento. La misma planteaba:"a. En la cátedra en la que Ud. trabaja, ¿se han incorporado materiales didácticos y/o bibliográficos vinculados a la perspectiva de género y mujeres? En caso afirmativo, ¿podría indicar en qué año, aproximadamente, ingresan éstos al Programa de la materia?".

En función de las cuarenta respuestas recibidas, obtuvimos treinta y cinco respuestas que responden de manera afirmativa mientras que las cinco restantes manifiestan no incluir. Estas últimas respuestas pertenecen a docentes de tres materias diferentes y dos de estas cinco respuestas aclaran que, si bien no han incorporado material específico, sí lo abordan en sus clases. Cabe aclarar, a su vez, que al menos un docente de las catorce ${ }^{\text {vii }}$ materias que comprende el bloque histórico obligatorio respondió el formulario. Es a partir de estos elementos, por ejemplo, que podemos comenzar a tejer algunas pistas desde las cuales interceptar los saberes que los docentes construyen, o van construyendo, desde la propia práctica. Así como matizar el análisis que uno puede realizar a partir de la observación del currículum estructural- formal. Por el contrario, dar lugar al currículum en acción, a través, en este caso, de las voces docentes nos permite acercarnos de otra manera a la historia enseñada.

En cuanto al año de ingreso de estas temáticas en las cátedras, indicamos un corte construido en función de las materias que abordan esta perspectiva antes y las que lo incluyen después de aprobarse el Plan de Estudios 2011. A su vez, en este primer agrupamiento, dos asignaturas las incorporan de manera precursora, nos referimos a Introducción a la Historia (integrantes de la cátedra indican el año 2000, aproximadamente) e Historia Americana I (aquí remiten a fines de los 80 y 90). De esta manera, afirmamos que estas dos materias fueron las que, pioneramente, introdujeron una historia investigada vinculada a género y mujeres. En el caso Historia General IV, estos tópicos aparecen influenciados a partir de los debates en torno a la protoindustria. En Historia General V, en las respuestas aparece señalado el 2007 como año tentativo de ingreso del tema mientras que, en Historia Americana II, se indica el año 2010, también, 
como fecha aproximada. Esta información revela que cinco cátedras incluyen estas temáticas, en el marco del Plan de Estudios 93, y que su inclusión precede al clamor social que se recrudece a partir del 2015. Asimismo, en la mayoría de estas cátedras, encontramos docentes que han realizado sus posgrados en el exterior, particularmente en EEUU, aunque son un grupo reducido de la planta general.

El segundo agrupamiento lo integran otras seis materias, es decir, las que incluyen estas temáticas luego de aprobarse el Plan de Estudios vigente (2011). Nos referimos a Historia Argentina II (las respuestas recabadas indican que cerca del 2013/2014 suman este tipo de materiales/bibliografía), posteriormente, podemos nombrar un bloque de cinco materias que sitúan este ingreso en el 2016: Introducción a la Problemática contemporánea, Prehistoria General y Americana, Historia General I (una de las respuestas, a su vez, referencia que en los teóricos se incorporan en el 2015), Historia General VI (no obstante, una de las respuestas señala que se aborda con anterioridad, quizás, al igual que la materia anterior, esto se produce previamente en los teóricos) e Historia Argentina I. Por lo anteriormente expuesto y según las respuestas obtenidas, sobre el total de estas catorce materias que dependen del Departamento de Historia, once materias (78\%) incluyen materiales didácticos y/o bibliográficos sobre género y mujeres. Si bien no se puede desconocer este significativo porcentaje, nos preguntamos qué lugar ocupan estas temáticas en dichas materias. Además, como matiz, señalamos que estos saberes, construidos en y desde la propia práctica, nos permiten identificar que estos temas se abordan en algunos casos, también, de manera "informal" en las clases.

\section{Consideraciones finales}

El relevo de las respuestas docentes nos permite indicar el año 2016 como un año bisagra, si se quiere, al ingresar significativamente estas temáticas dentro de la carrera (cinco cátedras más lo incorporan). Esto nos da pie para plantear que, en la década de los 90, imperó un currículum cargado de una fuerte impronta de sujetos históricos masculinos. $Y$, en ese sentido, tanto la historia investigada como la enseñada se encargaron de producirla y reproducirla. Hace unos años, en buena hora, las voces del pasado que levanta la historia enseñada comienzan a diversificarse, ganando terreno en la formación universitaria e iniciando un diálogo más fluido, interpelando de esta manera a la historia investigada. Por otra parte, señalamos que, más allá de establecer un acercamiento con estos temas, muchas veces su incorporación no logra conmover los marcos interpretativos existentes y hegemónicos. En algunas oportunidades, las mujeres ingresan nominalmente (lo que no es poco frente a semejante omisión) o ingresan en tanto "anexo" o a partir de "clases especiales". Si bien esto configura un piso de visibilidad, como esbozamos, en muchas oportunidades no logran disputar un análisis en términos de relaciones de género para reinterpretar en términos historiográficos y repensar ciertas periodicidades.

Bajo estas reflexiones y marcos teóricos nos preguntamos: ¿podríamos plantear que predomina un currículum que se encuentra en proceso de "des-colonización", ligado a una des-colonización de saberes y/o sujetxs hegemónicos? Retomando el planteo de Elizalde, Felitti y Queirolo (2009), ¿qué sucede con la diferencia sexual como categoría explicativa en la carrera? El relevo de las respuestas docentes nos permite identificar el año 2016 como un año en donde ingresan significativamente estas temáticas como contenidos dentro de la formación obligatoria de la carrera (cinco cátedras más lo incorporan), esto equivale a un $78 \%$, según las respuestas obtenidas. A su vez, e independientemente del momento en que se incorporan, lo cierto es que dichas inclusiones generan (según lo expresado por la mayoría de docentes) un reacomodamiento que obliga a des-pensar ciertas prácticas, contenidos, bibliografía para legitimar saberes (y prácticas) otras. ¿Podemos pensar, entonces, en un 
"desprendimiento" de los relatos hegemónicos? ¿Podemos plantear que, a partir de estas inclusiones, está surgiendo una transformación respecto de la historia que se enseña? Si bien se registran algunos cambios, por el momento, no podemos dimensionar en qué derivarán. No obstante, y como se expresa en uno de los formularios, la sola inclusión de algún que otro texto no implica una aprehensión de esta perspectiva en términos epistemológicos ni pedagógicos.

Si pensamos la formación, los contenidos y bibliografía estudiada hace diez o veinte años atrás, claramente podemos observar diferencias sustanciales respecto a la formación actual (como expresa un docente, "en historia todo está sujeto a revisión"), y retomando palabras de Feldman (2010), la selección de contenidos y bibliografía responde a decisiones de un determinado momento, sujetas a revisión.

Concluimos planteando que historizar e identificar cambios y permanencias dentro del Profesorado en Historia de la FaHCE nos permite resaltar la configuración del currículum en tanto artefacto cultural, histórico, situado y generizado. En suma, un currículum que si bien estuvo colonizado por sujetos masculino comienza a divisarse una transformación en clave de justicia curricular (Connell, 1997), inclusión y heterogeneidad.

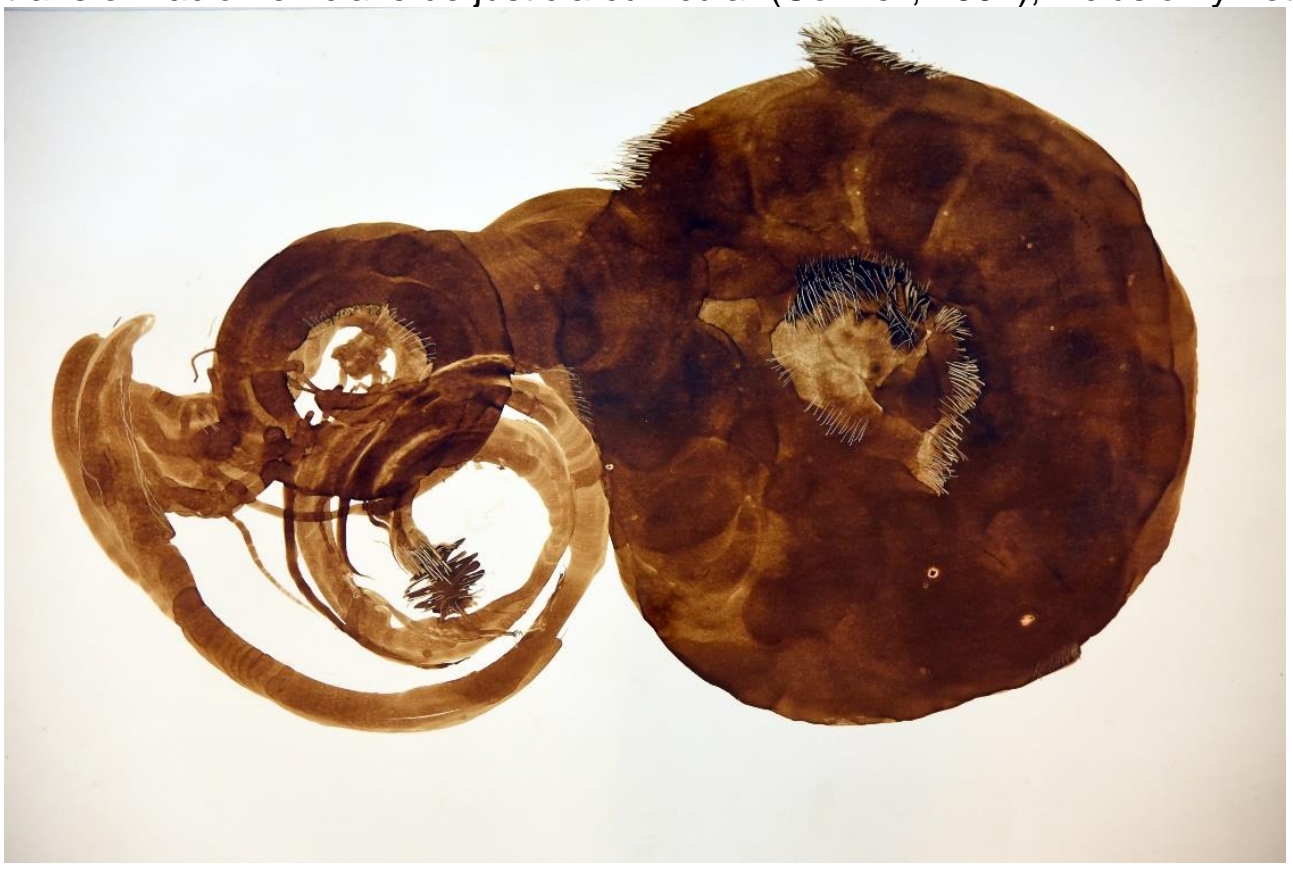

Panza, monocopias intervenida. Roberto Garro

\section{Notas}

'Se realizaron entrevistas a: Ana Julia Ramírez (actualmente se desempeña como Decana de la FaHCE. Al momento de realizarle la entrevista ocupaba el cargo de Secretaria Académica [20102014, 2014-2018]. Profesora Adjunta de la materia Historia General V, FaHCE. La entrevista se realizó en la FaHCE, el 11 de diciembre de 2017). Gonzalo de Amézola (Docente e investigador. Se desempeñó dentro de la FaHCE como Secretario Académico [1998-2001]. Fue director del Departamento de Historia [2003-2007]. Profesor Titular de la materia "Planificación Didáctica y Prácticas de la Enseñanza en Historia". La entrevista se realizó en un bar en Gonnet, el 5 de octubre de 2017). Juan Carnagui (Doctor en Historia. Secretario del Departamento de Historia [2010-2014, 2014-2018]. Profesor adjunto de Introducción a la problemática contemporánea. Investigador UNLP con lugar de trabajo en el Centro de Investigaciones Sociohistóricas [CISH]. La entrevista se realizó en la FaHCE, el 29 de noviembre de 2017). José Amícola (profesor desde 
1986 de la Facultad de Humanidades y Ciencias de la Educación, UNLP, en la cátedra de Introducción a la literatura de la Carrera de Letras. Se doctoró en Alemania en 1982 con una tesis sobre Roberto Arlt. La entrevista al profesor se realizó el 13 de octubre de 2017 por videollamada). María Luisa Femenías (profesora titular desde 1997 de la cátedra Antropología Filosófica, de la Facultad de Humanidades y Ciencias de la Educación, UNLP. Se doctoró en Filosofía, en la Universidad Complutense de Madrid. Fue directora del CInIG. Actual directora de la Especialización en Educación en Género y Sexualidades, FaHCE. La entrevista a la profesora María Luisa Femenías se realizó en un bar en CABA el 12 de octubre de 2017). Adriana Valobra (Doctora en Historia, directora del CInIG y profesora Titular de la materia Metodología I y Problemas de Historia Argentina - donde aborda temas de mujeres y género- de la carrera de Historia. Es investigadora de Conicet. La entrevista se realizó el 14 de diciembre de 2017 en su casa en La Plata). Silvia Mallo (docente e investigadora de la Facultad de Humanidades y Ciencias de la Educación, UNLP. Profesora Titular de la materia Historia Americana I de la carrera de Historia, desde 2002 a 2012. Dicha información la ha facilitado la oficina de Personal de la FaHCE. La entrevista se realizó en un bar en CABA el 20 de octubre de 2017). Gastón Guzmán (egresado de la carrera, miembro del Claustro Graduados de Junta asesora departamental de Departamento de Historia, la entrevista se realizó en un bar en La Plata, el 17 de octubre de 2017). Ada Ferrero (estudiante de la carrera de Historia, la entrevista se realizó 17 en la FaHCE, de octubre de 2017).

"Los programas se encuentran disponibles en Colección Archivo de programas de la FaHCE, http://www.memoria.fahce.unlp.edu.ar/

iiise reponen palabras entre corchetes para que puedan comprenderse los énfasis irónicos que agregó con su entonación la profesora.

iv Un tema que convoca el interés de docentes y estudiantes es el de la Educación Sexual Integral (ESI), sobre la que hay poca información sobre cómo llevarla adelante en el marco de las materias de estudio en secundaria. Una convocatoria que realizó graduados, en el mes de junio de 2017, en el marco del Espacio permanente de reflexión sobre la docencia en Historia, fue "Experiencias y desafíos en la aplicación de la Ley Nacional de Educación Sexual Integral (ESI) en las escuelas medias". En dicha oportunidad, expusieron como panelistas: Yamila Balbuena (Docente de la Cátedra Introducción a la Teoría Feminista y de Género- FaHCE/UNLP), Gisela Manzoni (Profesora de Historia, Capacitadora de ESI, Ex becaria doctoral CONICET, Doctoranda en Historia e Integrante del Centro Interdisciplinario de Investigaciones de Género [CInIG/FaHCE- UNLP]) y Flavia Tersigni (Docente y Capacitadora del programa Nacional de Educación Sexual Integral) sobre sus recorridos laborales y experiencias vinculadas a implementación de la Ley. Esta iniciativa supo congregar, además de una importante cantidad de asistentes, a diversos claustros, lo cual: "fue un encuentro con un saldo sumamente positivo, porque fueron muchas personas, pero además de perfiles diversos: estudiantes, graduados, docentes de la carrera, algunos de otras carreras; personas que a su vez habían perdido el vínculo con la Facultad, por tanto, se abrió muchísimo el público" (Fragmento de entrevista)

${ }^{\vee}$ Reglamento de Planes de Estudio. Ordenanza № 282/10. (Versión Taquigráfica Acta № 1197). Disponible

en: https://www.ing.unlp.edu.ar/sitio/institucional/legislacion/unlp/ordenanzas/282 reglamentacion plan es de estudios.pdf

${ }^{V i}$ Los contenidos mínimos del Plan 2010 (vigente en 2011) se encuentran disponibles en: http://www.memoria.fahce.unlp.edu.ar/planes/pl.94/pl.94.pdf, (pp. 9-19)

vii Se decidió exceptuar la materia Sociología General por pertenecer a otro Departamento.

\section{Bibliografía}

Abate, S. y Orellana, V. (2015). Notas sobre el currículum universitario. Prácticas profesionales y saberes en uso. Trayectorias Universitarias. Recuperado de:

https://revistas.unlp.edu.ar/TrayectoriasUniversitarias/article/view/2307/2252

Barrancos, D. (2005). Historia, historiografía y género. Notas para la memoria de sus vínculos en la Argentina. Aljaba, 9. Recuperado de:

http://www.scielo.org.ar/scielo.php?script=sci_arttext\&pid=S1669-57042005000100003

Barrancos, D. (2007) Mujeres en la sociedad argentina. Una historia de cinco siglos. Buenos Aires: Sudamericana. 
Birgin, A. (2012). Más allá de la capacitación. Debates acerca de la formación de los docentes en ejercicio. Buenos Aires: Paidós.

Bolla, L. y Rocha, M. (diciembre de 2018).Des-pensar para seguir pensando: saberes ¿otros? Mujeres y feminismo en los Programas universitarios de Historia y Filosofía. $X$ jornadas de Sociología. Facultad de Humanidades y Ciencias de la Educación, UNLP.

Burke, P. (2003). Formas de hacer Historia. Madrid: Alianza.

Connell, R. W. (1997). Escuelas y justicia social. Madrid: Morata.

Coscarelli, R. (2014). Currículo universitario y formación docente. En Morandi, G. y Ungaro, A. (Comps), La experiencia interpelada. Prácticas y perspectivas en la formación docente universitaria (pp. 95-108). La Plata: EDULP.

Coudannes Aguirre, M. (2010). La formación del profesor de historia en la universidad argentina. La creciente distancia entre investigación/ docencia y teoría/ práctica. Antítesis, 3 (6), 975-990. Recuperado de: http://www.uel.br/revistas/uel/index.php/antiteses/article/view/4579

Coudannes Aguirre, M. (2016). 20 años de Clío \& Asociados. La historia enseñada. Clío \& Asociados. La historia enseñada, 23, 15-25. Recuperado de:http://www.memoria.fahce.unlp.edu.ar/art_revistas/pr.8152/pr.8152.pdf

D'Antonio, D. (2012/2013). Género y clase: una mirada desde la historia social. REMS, Año 5/6, №5/6. Recuperado de:http://estudiosmaritimossociales.org/wp-content/uploads/2014/01/remsnc2ba-5-6-dossier-gc3a9nero-y-clase-presentacic3b3n-d-antonio-1.pdf

Da Silva, T. (1997). Descolonizar el currículum: estrategias para una pedagogía crítica. En Gentilli, P. (Comp.), Cultura, política y Currículo (Ensayos sobre la crisis de la escuela pública). (pp. 1-9) Buenos Aires: Losada.

Da Silva, T. (1999). Documentos de Identidad. Una introducción a las teorías del currículo. Belo Horizonte: Autêntica Editorial. Recuperado de:

http://www.fceia.unr.edu.ar/geii/maestria/DoraBibliografia/Ut.\%201/SILVA\%20docs\%20ident.pdf

Davini, M. C. (1995). La formación docente en cuestión: política y pedagogía. Buenos Aires: Paidós.

Davini, M. C. (1998). La formación docente en debate. Recuperado de:

https://cdn.educ.ar/dinamico/UnidadHtml get 2699e6d2-7a08-11e1-83d9-

ed15e3c494af/Curriculum Davini.pdf

Davini, M. C. (2015). La formación en la práctica docente. Buenos Aires: Paidós.

De Alba, A. (1998). Crisis, mito y perspectivas. Buenos Aires: Miño y Dávila.

De Amézola, G. A. (2015). La formación de profesores de Historia en las Universidades y las didácticas específicas ¿Hacia un nuevo debate?. Revista interdisciplinaria de formación docente, 1 (1), 47-57. Recuperado de:http://ppct.caicyt.gov.ar/index.php/kimun/article/view/7776/6971

De Lauretis, T. (2000). Diferencias: etapas de un camino a través del feminismo. Madrid: Horas y horas.

De Sousa Santo, B. (2010). Descolonizar el saber, reinventar el poder. Uruguay: Trilce Editorial. Recuperado

de:http://www.boaventuradesousasantos.pt/media/Descolonizar\%20el\%20saber_final\%20\%20C\%C3\%B3pia.pdf

Edelstein, G. (2014). Formar-se para la enseñanza en las universidades. Aportes desde experiencias en posgrado. En Morandi, G. yUngaro, A. (Comps), La experiencia interpelada. Prácticas y perspectivas en la formación docente universitaria (pp. 55-72). La Plata: EDULP.

Edelstein, G. (2015). Formar y formarse en la enseñanza. Buenos Aires: Paidós.

Elizalde, S., Felitti, K. y Queirolo, G. (2009). Género y sexualidades en las tramas del saber. Buenos Aires: Libros del Zorzal.

Feldman, D. (2010). Didáctica general. Buenos Aires: Ministerio de Educación de la Nación. Recuperado de: https://cedoc.infd.edu.ar/upload/Didactica_general.pdf

Felliti, K. y Queirolo, G. (2009). Historia. Cuerpos, género y sexualidades a través del tiempo. En Elizalde, S.,Felitti, K. y Queirolo, G.(Coord.) Género y sexualidades en las tramas del saber. Revisiones y propuestas. (pp. 27-57). Buenos Aires: Libros del Zorzal.

Flores, V. (mayo de 2015). ESI: Esa Sexualidad Ingobernable. El reto de des-heterosexualizar la pedagogía. Mesa: La escuela como productora de identidad: desafíos de una educación sexual integral no heteronormada. III Jornadas Interdisciplinarias de Géneros y Disidencia Sexual. Escuela 
Normal Superior №1. Recuperado de:http://escritoshereticos.blogspot.com.ar/2015/05/esi-esasexualidad-ingobernable-el-reto.html

Fox Keller, E. (1991). Reflexiones sobre género y ciencia. Valencia: Edicions Alfons El Magnànim. Gamba, S. (Coord). (2009). Diccionario de estudios de género y feminismos. Buenos Aires: Biblos. Haraway, D. (1995). Ciencias, cyborgs y mujeres. La invención de la naturaleza. Madrid: Sudakuir. Harding, S. (1996). Ciencia y feminismo. Madrid: Ediciones Morata.

Hobsbawm, E. (1987). El hombre y la mujer: imágenes a la izquierda. En: Hobsbawm, E. El mundo del trabajo. Estudios históricos sobre la formación y la revolución de la clase obrera (pp. 117-143). Barcelona: Crítica.

Jackson, P. W. (2002). Prácticas de la enseñanza. Buenos Aires: Amorrortu.

Le Goff, J. (1991). Pensar la Historia. Modernidad, presente y progreso. Barcelona: Paidós.

Lobato, M. (2010). Historia del trabajo: género y clase. En Cernadas, J. y Lvovich, D. (Comps), Historia, ¿para qué? Revisitas a una vieja pregunta (pp. 121-140). Buenos Aires: Prometeo. Lopes Louro, G. (2012). "Extrañar" el currículum. En Spadaro, M. C. (comp.) Enseñar filosofía, hoy (pp. 109-120). La Plata: Universidad Nacional de La Plata.

Maestro Gonzalez, P. (1997). Historiografía, didáctica y enseñanza de la historia (la concepción de la Historia enseñada). Clío y asociados. La historia enseñada, (2), 9-34.

Maffia, D. (2007). Epistemología feminista: la subversión semiótica de las mujeres en la ciencia. Revista Venezolana de Estudios de la mujer, 12 (12). Recuperado de: http://cemucv.org.ve/?page_id=22

Manzoni, G. (2011). Huellas de mujeres entre dos siglos o cómo comenzar, algún día, con la historia de las mujeres en el aula. Clío \& Asociados, (15), 137-152. Recuperado

de:http://www.memoria.fahce.unlp.edu.ar/library?a=d\&c=arti\&d=Jpr5018

Morgade, G. (2018). Las Universidades Públicas como territorios del patriarcado. Política Universitaria. La universidad hoy, a 100 años de la Reforma. Vol 1, 5, Especial mayo Recuperado de: https://conadu.org.ar/wp-content/uploads/5-ESPECIAL-WEB.pdf

Palermo, Z. (2014). Para una pedagogía decolonial. Ciudad Autónoma de Buenos Aires: Del Signo. Plá, S. (2012). La enseñanza de la historia como objeto de investigación. Secuencia, 84, 163-184. Recuperado de: http://www.redalyc.org/pdf/3191/319128360007.pdf

Pateman, C. (1995). El contrato sexual. México, Universidad Autónoma Metropolitana: Anthropos.

Rocha, M. (2017). La historia del género y el género en historia. Apuntes preliminares dentro del profesorado en Historia, de la Universidad Nacional de La Plata. Revista Clío y Asociados, (25), 8697. Recuperado de: http://www.memoria.fahce.unlp.edu.ar/art_revistas/pr.8627/pr.8627.pdf

Scott, J. (1996). El género, una categoría útil para el análisis histórico. En Lamas, M. (Comp.), El género: la construcción cultural de la diferencia sexual (pp. 265-302). México: PUEG.

Scott, J. (2003). Historia de las mujeres. En Burke, P. (Comp.), Formas de hacer Historia (pp. 5988). Madrid: Alianza.

Sharpe, J. (2003). Historia desde abajo. En Burke, P. (Comp.), Formas de hacer Historia (pp. 3958). Madrid: Alianza.

Stone, L. y Hobsbawm, E. (1983). La historia como narrativa. Débats, 4, 91-110.

Valobra, A. (2005). Algunas consideraciones acerca de la relación entre historia de las mujeres y género en Argentina. Nuevo Topo. (1), 101-122.

Zavala, A. (2014). Y entonces, ¿la historia enseñada qué es? Reflexiones en torno a las relaciones entre lo que sabemos y lo que enseñamos. Revista Clío y Asociados, (18-19), 11-40. Recuperado de: http://bibliotecavirtual.unl.edu.ar/publicaciones/index.php/ClioyAsociados/article/view/4735/7227 Programa referenciado

Mallo, S. C. (1989). La mujer en el período colonial (Programa). UNLP, Dpto. de Historia. Caja 1989-B-76 (primer piso de BIBHUMA, sector Referencia). 